\title{
Motion induced by asymmetric excitation of the quantum vacuum
}

\author{
Jeferson Danilo L. Silva, ${ }^{1, *}$ Alessandra N. Braga, ${ }^{2, \dagger}$ Andreson L. C. Rego,,${ }^{3, \dagger}$ and Danilo T. Alves ${ }^{4, \S}$ \\ ${ }^{1}$ Campus Salinópolis, Universidade Federal do Pará, 68721-000, Salinópolis, Brazil \\ ${ }^{2}$ Instituto de Estudos Costeiros, Universidade Federal do Pará, 68600-000, Bragança, Brazil \\ ${ }^{3}$ Instituto de Aplicação Fernando Rodrigues da Silveira, \\ Universidade do Estado do Rio de Janeiro, 20261-232, Rio de Janeiro, Brazil \\ ${ }^{4}$ Faculdade de Física, Universidade Federal do Pará, 66075-110, Belém, Brazil
}

(Dated: September 22, 2020)

\begin{abstract}
During the last fifty-one years, the effect of excitation of the quantum vacuum field induced by its coupling with a moving object has been systematically studied. Here, we propose and investigate a somewhat inverted setting: an object, initially at rest, whose motion becomes induced by an excitation of the quantum vacuum caused by the object itself. In the present model, this excitation occurs asymmetrically on different sides of the object by a variation in time of one of its characteristic parameters, which couple it with the quantum vacuum field.
\end{abstract}

\section{INTRODUCTION}

In 1969, Gerald T. Moore published in his $\mathrm{PhD}$ thesis the prediction that a mirror in movement can excite the quantum vacuum, generating photons [1]. This effect is nowadays known as the dynamical Casimir effect (DCE) and was investigated, during the 1970 s, in other pioneering articles by DeWitt [2], Fulling and Davies [3, 4], Candelas and Deutsch [5], among others. Since then, many other authors have dedicated to investigate the DCE (some excellent reviews on the DCE can be found in Ref. [6-9]).

In his pioneering work, Moore remarked that "to practical experimental situations, the creation of photons from the zero-point energy is altogether negligible" [1]. In an attempt to overcome this difficulty, several ingenious proposals have been made aiming to observe the particle creation from vacuum by experiments based on the mechanical motion of a mirror [10-15], but this remains as a challenge [9]. However, the particle creation from the vacuum occurs, in general, when a quantized field is submitted to time-dependent boundary conditions, with moving mirrors being a particular case. Therefore, it is not necessary to move a mirror to generate real particles from the vacuum. Within this more general view, alternative ways to detect particle creation from vacuum were inspired in the ideas of Yablonovitch [16] and Lozovik et al. [17], which consist in exciting the vacuum field by means of time-dependent boundary conditions imposed to the field by a motionless mirror whose internal properties rapidly vary in time. In this context, Wilson et al. [18] observed experimentally the particle creation from vacuum, using a time-dependent magnetic flux applied in a coplanar waveguide (transmission line) with a superconducting quantum interference device (SQUID) at one of the extremities, changing the inductance of the SQUID, and thus yielding a time-dependent boundary condition [18]. Other experiments

\footnotetext{
*jdanilo@ufpa.br

† alessandrabg@ufpa.br

¥ andresonlcr@gmail.com

$\S$ danilo@ufpa.br
}

have also been done [19-21], and other have been proposed [22-31].

When one moves an object imposing changes in the vacuum field, the latter offers resistance, extracting kinetic energy from the object, which is converted into real particles. In this case, one can say that the net action of the vacuum is against the motion. Here, we propose and investigate a somewhat inverted situation: an object, initially at rest, isolated from everything and just interacting with the quantum vacuum, whose motion becomes induced by an excitation of the vacuum field caused by the object itself. In other words, we are looking for a situation where the vacuum field acts in favor of a motion in a preferred direction. With this in mind, we propose a model where an object imposes a change to the quantum field by the time variation of the properties of the object. Resisting to this change, the vacuum field extracts energy from the object, exciting the quantum field and converting the energy into real particles. A fundamental point of the model presented here is that, to get in motion in a preferred direction, the object has to excite the quantum vacuum differently on each side, which requires that an asymmetry must be introduced in the object. Taking into account the same simplified one-dimensional model considered in the pioneering articles on the DCE [1-5], we consider the coupling of a static point object with a quantum real scalar field in $(1+1) \mathrm{D}$ via a $\delta-\delta^{\prime}$ potential, which simulates a partially reflecting object with asymmetric scattering properties on each side [32, 33]. When the coupling parameters vary in time, this model simulates an object exciting asymmetrically the fluctuations of the quantum vacuum, which produces a non-null mean force acting on the object, so that it can get in motion. Then, instead of against, the vacuum acts in favor of the motion in a preferred direction. But, not completely in favor, since, once in motion, a dynamical Casimir force acts on the object, so that part of its kinetic energy is extracted by the vacuum fluctuations and goes to the field.

\section{THE INITIAL MODEL}

We are interested in an object whose interaction with the field is described by an asymmetric scattering matrix, intend- 
ing to excite asymmetrically the quantum vacuum fluctuations. The interaction between the object and the field described by a Dirac $\delta$ potential produces a (left-right) symmetric scattering matrix $[34,35]$. Therefore, to generate an asymmetry, we also consider the presence of an odd $\delta^{\prime}$ term in the description of the interaction, so that our starting point is the following lagrangian for a real massless scalar field in $(1+1) \mathrm{D}$ :

$$
\mathcal{L}=\mathcal{L}_{0}-\left[\mu(t) \delta(x)+\lambda_{0} \delta^{\prime}(x)\right] \phi^{2}(t, x),
$$

where $\mathcal{L}_{0}=\left[\partial_{t} \phi(t, x)\right]^{2}-\left[\partial_{x} \phi(t, x)\right]^{2}$ is the lagrangian for the free field, and the real parameters $\lambda_{0}$ and $\mu$, together with $\delta$ and $\delta^{\prime}$ functions, describe the coupling between the quantum field and a static object located at the point $x=0$. These coupling parameters are related to the properties of the object. The parameter $\mu$ is a prescribed function of time: $\mu(t)=\mu_{0}[1+\epsilon f(t)]$, where $\mu_{0} \geq 0$ is a constant, $f(t)$ is an arbitrary function such that $|f(t)| \leq 1$ and $\epsilon \ll 1$. In this way, the parameter $\mu$ is a perturbation in time around the value $\mu_{0}$. We consider that this time variation of $\mu$ occurs at the expense of the internal energy of the object, with null heat transfer between the object and the environment. As we discuss next, the point object described by this model is partially reflective, so that a modification in the parameter $\mu$ implies a change in the transmission and reflection coefficients. Hereafter, we consider that $c=\hbar=1$, tilde indicates the Fourier transform, and the subscript $+(-)$ indicates the right (left) side.

The field equation for this model is $\left(\partial_{t}^{2}-\partial_{x}^{2}\right) \phi(t, x)+$ $2\left[\mu(t) \delta(x)+\lambda_{0} \delta^{\prime}(x)\right] \phi(t, x)=0$. It will be convenient to split the field as $\phi(t, x)=\Theta(x) \phi_{+}(t, x)+\Theta(-x) \phi_{-}(t, x)$, where $\Theta(x)$ is the Heaviside step function and the fields $\phi_{+}$and $\phi_{-}$are the sum of two freely counterpropagating fields, namely $\phi_{+(-)}(t, x)=\varphi_{\text {out (in) }}(t-x)+\psi_{\text {in(out) }}(t+$ $x)$, where the labels "in" and "out" indicate, respectively, the incoming and outgoing fields with respect to the object (see Fig. 1.a). In terms of the Fourier transforms, we can write $\phi_{+(-)}(t, x)=\int \frac{\mathrm{d} \omega}{2 \pi} \tilde{\phi}_{+(-)}(\omega, x) \mathrm{e}^{-i \omega t}$, where $\tilde{\phi}_{+(-)}(\omega, x)=\tilde{\varphi}_{\text {out }(\text { in })}(\omega) \mathrm{e}^{i \omega x}+\tilde{\psi}_{\text {in }(\text { out })}(\omega) \mathrm{e}^{-i \omega x}$. From the field equation, we get the matching conditions $\tilde{\phi}\left(\omega, 0^{+}\right)=$ $\left[\left(1+\lambda_{0}\right) /\left(1-\lambda_{0}\right)\right] \tilde{\phi}\left(\omega, 0^{-}\right)$and $\partial_{x} \tilde{\phi}\left(\omega, 0^{+}\right)=\left[\left(1-\lambda_{0}\right) /(1+\right.$ $\left.\left.\lambda_{0}\right)\right] \partial_{x} \tilde{\phi}\left(\omega, 0^{-}\right)+\left[2 /\left(1-\lambda_{0}^{2}\right)\right] \int \frac{\mathrm{d} \omega^{\prime}}{2 \pi} \tilde{\mu}\left(\omega-\omega^{\prime}\right) \tilde{\phi}\left(\omega^{\prime}, 0^{-}\right)$. After an algebraic manipulation in these equations, we obtain

$$
\begin{aligned}
\Phi_{\text {out }}(\omega)= & S_{0}(\omega) \Phi_{\text {in }}(\omega)+\int \frac{\mathrm{d} \omega^{\prime}}{2 \pi} \delta S_{1}\left(\omega, \omega^{\prime}\right) \Phi_{\text {in }}\left(\omega^{\prime}\right) \\
& +\int \frac{\mathrm{d} \omega^{\prime}}{2 \pi} \int \frac{\mathrm{d} \omega^{\prime \prime}}{2 \pi} \delta S_{2}\left(\omega, \omega^{\prime}, \omega^{\prime \prime}\right) \Phi_{\text {in }}\left(\omega^{\prime \prime}\right),
\end{aligned}
$$

with

$\Phi_{\text {out }(\mathrm{in})}(\omega)=\left(\begin{array}{c}\tilde{\varphi}_{\text {out }(\mathrm{in})}(\omega) \\ \tilde{\psi}_{\mathrm{out}(\mathrm{in})}(\omega)\end{array}\right), S_{0}(\omega)=\left(\begin{array}{ll}s_{+}(\omega) & r_{+}(\omega) \\ r_{-}(\omega) & s_{-}(\omega)\end{array}\right)$,

where $S_{0}(\omega)$ is the scattering matrix, with $s_{ \pm}(\omega)=\omega(1-$ $\left.\lambda_{0}^{2}\right) /\left[i \mu_{0}+\omega\left(1+\lambda_{0}^{2}\right)\right]$ and $r_{ \pm}(\omega)=-\left(i \mu_{0} \mp 2 \omega \lambda_{0}\right) /\left[i \mu_{0}+\right.$ $\left.\omega\left(1+\lambda_{0}^{2}\right)\right]$ being the transmission and reflection coefficients, respectively. Notice that the change $\lambda_{0} \leftrightarrow-\lambda_{0}$ leads to $r_{+}(\omega) \leftrightarrow r_{-}(\omega)$, i.e. the object shifts its properties from one side to the other. Moreover, $S_{0}(\omega)$ is analytic for $\operatorname{Im} \omega>0$ (as required by causality $[36,37]$ ), unitary and real in the temporal domain. The terms $\delta S_{1}\left(\omega, \omega^{\prime \prime}\right)$ and $\delta S_{2}\left(\omega, \omega^{\prime}, \omega^{\prime \prime}\right)$ represent the first-order and second-order corrections to $S_{0}(\omega)$ due to the time-dependence of $\mu$ via $f(t)$. They are given by $\delta S_{1}\left(\omega, \omega^{\prime}\right)=\epsilon \alpha\left(\omega, \omega^{\prime}\right) \mathbb{S}\left(\omega^{\prime}\right)$ and $\delta S_{2}\left(\omega, \omega^{\prime}, \omega^{\prime \prime}\right)=$ $\epsilon^{2} \alpha\left(\omega, \omega^{\prime}\right) \alpha\left(\omega^{\prime}, \omega^{\prime \prime}\right) \mathbb{S}\left(\omega^{\prime \prime}\right)$, where $\alpha\left(\omega, \omega^{\prime}\right)=-i \mu_{0} \tilde{f}(\omega-$ $\left.\omega^{\prime}\right) /\left[i \mu_{0}+\omega\left(1+\lambda_{0}^{2}\right)\right]$ and

$$
\mathbb{S}(\omega)=\left(\begin{array}{cc}
s_{+}(\omega) & 1+r_{+}(\omega) \\
1+r_{-}(\omega) & s_{-}(\omega)
\end{array}\right) .
$$

Particularly, $\mu_{0} \rightarrow \infty$ leads to the case of a perfectly reflecting object $\left[s_{ \pm}(\omega) \rightarrow 0\right]$ imposing to the field the Dirichlet boundary condition in both sides, for which $\delta S_{1} \rightarrow 0$ and $\delta S_{2} \rightarrow 0$, recovering the configuration of a perfectly reflecting object whose properties do not vary in time. On the other hand, the limit $\lambda_{0} \rightarrow 1\left(\lambda_{0} \rightarrow-1\right)$ also leads to a perfectly reflecting object, but imposing to the field the Dirichlet and Robin (Robin and Dirichlet) boundary conditions at the left and right sides of the object, respectively.

(a)

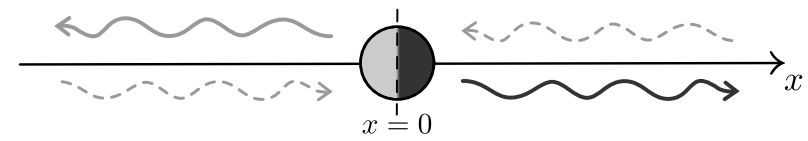

(b)

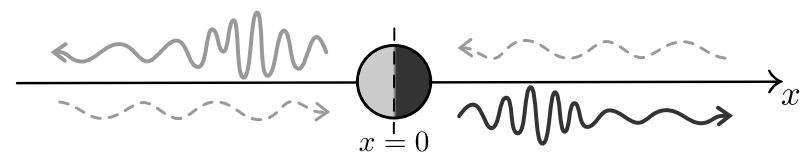

(c)

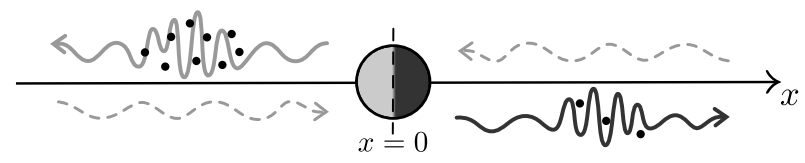

Figure 1. Illustration of the excitation of the quantum vacuum by an object fixed at $x=0$, whose asymmetry in its scattering matrix is represented by its two faces in gray and black. The dashed wavy lines represent the unperturbed "in" fields $\varphi_{\text {in }}(t-x)$ (left) and $\psi_{\text {in }}(t+x)$ (right). (a) Object for $t<-\tau(\tau>0)$, when $\mu(t) \approx \mu_{0}$. The solidgray and solid-dark wavy lines represent the unperturbed "out" fields $\psi_{\text {out }}(t+x)$ (left) and $\varphi_{\text {out }}(t-x)$ (right), respectively. (b) Object at an instant $t$, with its parameter $\mu$ varying in time. The irregular parts of the solid wavy lines represent the perturbed parts of the "out" fields $\psi_{\text {out }}(t+x)$ and $\varphi_{\text {out }}(t-x)$, respectively. (c) The object for $t>\tau$, when $\mu(t) \approx \mu_{0}$, and we calculate the number of created particles (represented by the dark points). Note that we have more particles produced in the left side.

\section{SPECTRUM, ENERGY AND MOMENTUM}

Let us consider the initial situation $(t<-\tau)(\tau>0)$ when the characteristic parameters of the object are constant $\left[\lambda_{0}\right.$ and $\left.\mu(t<-\tau) \approx \mu_{0}\right]$ and the state of the field is the quantum vacuum (see Fig. 1.a). At a certain instant $-\tau$, the properties of the object start to vary $\left[\mu_{0} \rightarrow \mu(t)\right]$, changing the boundary conditions imposed to the field, exciting the fluctuations of the quantum vacuum in the interval 
$-\tau<t<\tau$ (see Fig. 1.b). The final situation $(t>\tau)$ is when the object recovers its constant characteristic parameters $\left[\lambda_{0}\right.$ and $\left.\mu(t>\tau) \approx \mu_{0}\right]$ and real particles are created (see Fig. 1.c). The spectrum of created particles can be computed by $n(\omega)=2 \omega \operatorname{Tr}\left\langle 0_{\text {in }}\left|\Phi_{\text {out }}(-\omega) \Phi_{\text {out }}^{\mathrm{T}}(\omega)\right| 0_{\text {in }}\right\rangle$ [37]. From Eq. (2), calculated at order up to $\mathcal{O}\left(\epsilon^{2}\right)$, we have that $n(\omega)=n_{+}(\omega)+n_{-}(\omega)$, where

$$
n_{ \pm}(\omega)=\frac{\epsilon^{2}}{2 \pi^{2}}\left(1 \pm \lambda_{0}\right)^{2}\left(1+\lambda_{0}^{2}\right) \int_{0}^{\infty} \mathrm{d} \omega^{\prime} \eta\left(\omega, \omega^{\prime}\right),
$$

with $\eta\left(\omega, \omega^{\prime}\right)=\Upsilon(\omega) \Upsilon\left(\omega^{\prime}\right)\left|\tilde{f}\left(\omega+\omega^{\prime}\right)\right|^{2}$ and $\Upsilon(\omega)=$ $\mu_{0} \omega /\left[\mu_{0}^{2}+\omega^{2}\left(1+\lambda_{0}^{2}\right)^{2}\right]$. Therefore, we get $n_{-}(\omega)=$ $\left[\left(1-\lambda_{0}\right) /\left(1+\lambda_{0}\right)\right]^{2} n_{+}(\omega)$, which means that the spectrum for one side of the object differs from the other one by a frequency-independent global factor. For $\lambda_{0}>0\left(\lambda_{0}<0\right)$ $n_{-}(\omega)$ is smaller (greater) than $n_{+}(\omega)$.

The total number of created particles is given by $\mathcal{N}=$ $\int_{0}^{\infty} \mathrm{d} \omega n(\omega)$, and the number in each side of the object is $\mathcal{N}_{ \pm}=\int_{0}^{\infty} \mathrm{d} \omega n_{ \pm}(\omega)$, so that we can write $\mathcal{N}_{-}=$ $\left[\left(1-\lambda_{0}\right) /\left(1+\lambda_{0}\right)\right]^{2} \mathcal{N}_{+}$. Note that $\mathcal{N}$ is greater in the right side of the object if $\lambda_{0}>0$ and smaller if $\lambda_{0}<0$ (this latter case is illustrated in Fig. 1.c). Particularly, for the perfectly reflecting cases where $\lambda_{0}=1\left(\lambda_{0}=-1\right)$, we get $\mathcal{N}_{-}=0$ $\left(\mathcal{N}_{+}=0\right)$, so that the particles are created only in one side of the object.

The energy and momentum of the created particles in each side are given, respectively, by $\mathcal{E}_{ \pm}=\int_{0}^{\infty} \mathrm{d} \omega \omega n_{ \pm}(\omega)$ and $\mathcal{P}_{ \pm}= \pm \mathcal{E}_{ \pm}$. The total energy $\mathcal{E}$ and momentum $\mathcal{P}$ are $\mathcal{E}=$ $\mathcal{E}_{+}+\mathcal{E}_{-}$and $\mathcal{P}=\mathcal{P}_{+}+\mathcal{P}_{-}$. Specifically,

$$
\mathcal{P}=\frac{2 \epsilon^{2}}{\pi^{2}} \lambda_{0}\left(1+\lambda_{0}^{2}\right) \int_{0}^{\infty} \mathrm{d} \omega \int_{0}^{\infty} \mathrm{d} \omega^{\prime} \omega \eta\left(\omega, \omega^{\prime}\right),
$$

which is negative for $\lambda_{0}<0$ and positive for $\lambda_{0}>0$. Then, a static object, initially fixed at $x=0$, with its properties varying in time, can excite asymmetrically the fluctuations of the quantum vacuum, generating into the field a net momentum $\mathcal{P} \neq 0$. For instance, for the perfectly reflecting case where $\lambda_{0}=1\left(\lambda_{0}=-1\right)$, we obtain $\mathcal{P}_{-}=0\left(\mathcal{P}_{+}=0\right)$, so that momentum is transferred to the field (by exciting it) just in one of the sides of the object. This net momentum implies in a net force acting on the object.

\section{FORCE ON THE STATIC OBJECT}

Let us now obtain the expression for the mean force acting on the object at $x=0$ due to the field fluctuations (see Fig. 1.b). The components of the energy-momentum tensor for a scalar field in $1+1$ dimensions are given by $T_{00}=T_{11}=\left[\varphi^{\prime}(t-x)\right]^{2}+\left[\psi^{\prime}(t+x)\right]^{2} \equiv E(t, x)$, and $T_{01}=T_{10}=\left[\varphi^{\prime}(t-x)\right]^{2}-\left[\psi^{\prime}(t+x)\right]^{2} \equiv P(t, x)$, where $E(t, x)$ and $P(t, x)$ are the energy and momentum densities respectively, and their mean values can be written as $\left\langle E_{j}(t, x)\right\rangle=\operatorname{Tr}\left[\partial_{t} \partial_{t^{\prime}}\left\langle\Phi_{j}(t, x) \Phi_{j}^{\mathrm{T}}\left(t^{\prime}, x\right)\right\rangle\right]_{t=t^{\prime}}$ and $\left\langle P_{j}(t, x)\right\rangle$ $=\operatorname{Tr}\left[\operatorname{diag}(1,-1) \partial_{t} \partial_{t^{\prime}}\left\langle\Phi_{j}(t, x) \Phi_{j}^{\mathrm{T}}\left(t^{\prime}, x\right)\right\rangle\right]_{t=t^{\prime}}$, where $j=$ out, in. The force acting on the object due to the field fluctuations can be found as the difference between the radiation pressure $\left(T_{11}\right)$, on the left and on the right sides of the object. Therefore, the mean force is given by $F_{\mu}(t)=$ $\left\langle P_{\text {in }}(t, 0)-P_{\text {out }}(t, 0)\right\rangle$ and it can be written as

$$
F_{\mu}(t) \approx F_{\mu}^{(1)}(t) \epsilon+F_{\mu}^{(2)}(t) \epsilon^{2} .
$$

Taking its Fourier transform and considering Eq. (2), we obtain $\tilde{F}_{\mu}(\omega) \approx \tilde{F}_{\mu}^{(1)}(\omega) \epsilon+\tilde{F}_{\mu}^{(2)}(\omega) \epsilon^{2}$, where the mean value was taken considering a vacuum as the initial state of the field. The first-order term is $\tilde{F}_{\mu}^{(1)}(\omega)=\chi_{1}(\omega) \tilde{f}(\omega)$ where

$\chi_{1}(\omega)=\frac{2 \lambda_{0} \omega^{2}}{\pi \rho^{2}\left(\lambda_{0}^{2}+1\right)}\left\{\frac{\rho+i}{\rho+2 i}\left[2 i \arctan \rho-\ln \left(\rho^{2}+1\right)\right]-i \rho\right\}$,

and $\rho=\left(\lambda_{0}^{2}+1\right) \omega / \mu_{0}$. The second-order term is $\tilde{F}_{\mu}^{(2)}(\omega)=$ $\int \mathrm{d} \omega^{\prime} \int \mathrm{d} \omega^{\prime \prime} \chi_{2}\left(\omega, \omega^{\prime}, \omega^{\prime \prime}\right) \alpha\left(\omega^{\prime},-\omega^{\prime \prime}\right) \alpha\left(\omega-\omega^{\prime \prime}, \omega^{\prime}\right)$ where

$$
\begin{aligned}
\chi_{2}\left(\omega, \omega^{\prime}, \omega^{\prime \prime}\right)= & \lambda_{0}\left[h\left(-\omega^{\prime}\right) \Theta\left(\omega^{\prime \prime}\right) \omega^{\prime 2}\left(1+\lambda_{0}^{2}\right)-\omega^{\prime \prime} / 2\right] \\
& \times h\left(\omega^{\prime \prime}\right)\left(\omega^{\prime \prime}-\omega\right) \operatorname{sgn}\left(\omega^{\prime \prime}\right) / \pi^{2},
\end{aligned}
$$

and $h(\omega)=1 /\left[i \mu_{0}+\omega\left(1+\lambda_{0}^{2}\right)\right]$.

\section{FREE TO MOVE}

So far, the object has been assumed to be fixed at $x=0$, as described by the langrangian (1). Now, let us consider that for $t<-\tau$ the object is kept at $x=0$ (Fig. 2.a), but for $t>-\tau$ it is free to move. Even if $\mu(t)=\mu_{0}$ for $t>-\tau$, a fluctuating force from the quantum vacuum field would act on the object, so that it would start a Brownian motion [38-41]. On the other hand, one can use one of the degrees of freedom of the model, namely the initial mass of the object $\left(M_{0}\right)$, to simplify the problem. Assuming $M_{0}$ sufficiently large, the mean-squared displacement in the position of the object, during the interval $-\tau<t<\tau$, can be neglected and, therefore, the object remains at $x \approx 0$ in the mentioned interval.

Now, let us consider again $\mu(t)=\mu_{0}[1+\epsilon f(t)]$ in the interval $-\tau<t<\tau$ (Fig. 2.b), with $M_{0}$ remaining large enough to the Brownian motion be neglected, and the object free to move for $t>-\tau$ (Fig. 2.c). For this case, the boundary condition imposed to the field on the static object at $x=0$ must now be replaced by a boundary condition considered in the instantaneous position $x=q(t)$ of the moving object, observed from the point of view of an inertial frame where the object is instantaneously at rest (called tangential frame), and then be mapped into a boundary condition viewed by the laboratory system [33, 36, 42-44]. This means that the force $F_{\mu}(t)$ [Eq. (6)] needs to be replaced by a modified $F_{\mu q}(t, \dot{q}(t))$, which now can depend on the velocity of the object [for consistency, we consider that $\left.F_{\mu q}(t, 0)=F_{\mu}(t)\right]$. In addition to the force $F_{\mu q}(t, \dot{q}(t))$, the motion of the object gives rise to an extra disturbance to the vacuum field, from which arises an additional (dynamical Casimir) force acting on the object. This force is here represented by $F_{q}\left(\partial_{t}^{3} q(t), \partial_{t}^{4} q(t), \ldots\right)$, so that it acts on non-uniformly accelerating objects (see, for instance, Ref. $[3,45])$. For consistency, we assume that, for a static object, 
$F_{q}(0,0, \ldots)=0$. As an example, if one considers, for instance $\lambda_{0} \rightarrow-1$, the object imposes to the field on the left (right) side the Robin (Dirichlet) boundary condition. For this case, $F_{q}\left(\partial_{t}^{3} q(t), \partial_{t}^{4} q(t), \ldots\right) \approx 1 /(6 \pi) \partial_{t}^{3} q(t)-1 /\left(6 \pi \mu_{0}\right) \partial_{t}^{4} q(t)$ $[36,45,46]$. In summary, two forces act on the object free to move, a force $F_{\mu q}(t, \dot{q}(t))$ (related to the time-varying properties of the object) and a force $F_{q}\left(\partial_{t}^{3} q(t), \partial_{t}^{4} q(t), \ldots\right)$ (related to the motion of the object).

(a)

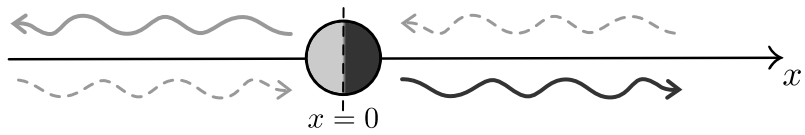

(b)

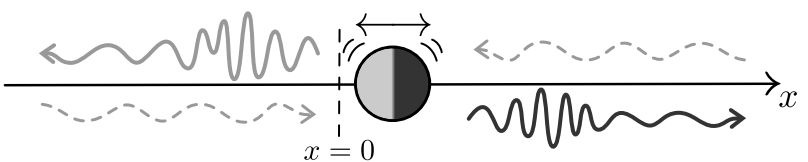

(c)

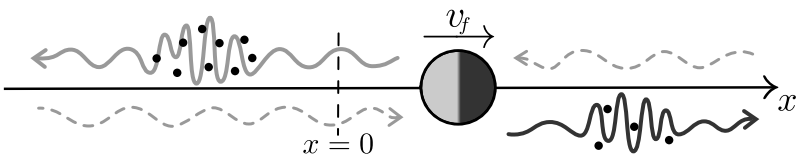

Figure 2. Illustration of an object, initially at rest (a) but free to move [(b) and (c)], whose motion becomes induced by an excitation of the quantum vacuum caused by the object itself. The object, characterized by an asymmetric scattering matrix, is represented by a circle with two faces in gray and black. The dashed wavy lines represent the unperturbed "in" fields $\varphi_{\text {in }}(t-x)$ (left) and $\psi_{\text {in }}(t+x)$ (right). (a) The object for $t<-\tau$, when $\mu(t) \approx \mu_{0}$. The solidgray and solid-dark wavy lines represent the unperturbed "out" fields $\psi_{\text {out }}(t+x)$ (left) and $\varphi_{\text {out }}(t-x)$ (right), respectively. (b) The object at an instant $t$, when the parameter $\mu$ is varying in time. The irregular parts of the solid-gray and of the solid-dark wavy lines represent the perturbed parts of the "out" fields $\psi_{\text {out }}(t+x)$ and $\varphi_{\text {out }}(t-x)$, respectively. Under this situation, the object is subjected to the force $F_{\mu q}^{(1)}(t, \dot{q}(t)) \epsilon+F_{\mu q}^{(2)}(t, \dot{q}(t)) \epsilon^{2}+F_{q}\left(\partial_{t}^{3} q(t), \partial_{t}^{4} q(t), \ldots\right)$ and gets in motion. (c) The object for $t>\tau$, when $\mu(t) \approx \mu_{0}$, and we calculate the number of created particles (represented by the dark points), moving with a final constant mean velocity $v_{f}$. Note that we have more particles produced in the left side, coinciding in this illustration with a larger flux of particle momentum.

From the energy conservation, and assuming that the excitation of the quantum vacuum occurs at the expense of the energy of the object [so that its initial mass $M_{0}$ becomes time-dependent: $\left.M_{0} \rightarrow M(t)\right]$, we have $M(t) \approx M_{0}[1-$ $\left.\mathcal{E}_{\text {field }}(t) / M_{0}\right] /\left[1+\dot{q}(t)^{2} / 2\right]$, where $\mathcal{E}_{\text {field }}(t)$ is the energy stored in the field, and the velocities of the object are considered non-relativistic (remember that $c=1$ ). We consider, now, the approximation $\mathcal{E}_{\text {field }}(t) / M_{0} \ll 1$, which means that the energy $\mathcal{E}_{\text {field }}(t)$ is negligible if compared to the initial energy of the object. We also consider the non-relativistic assumption $\dot{q}(t)^{2} \ll 1$. Then we have $M(t) \approx M_{0}$.

Considering all forces acting on the object, we have the equation of motion $F_{\mu q}(t, \dot{q}(t))+F_{q}\left(\partial_{t}^{3} q(t), \partial_{t}^{4} q(t), \ldots\right) \approx$ $M_{0} \ddot{q}(t)$. We also consider that, up to second order in $\epsilon$, the force $F_{\mu q}(t, \dot{q}(t))$ can be written as $F_{\mu q}(t, \dot{q}(t)) \approx$ $F_{\mu q}^{(1)}(t, \dot{q}(t)) \epsilon+F_{\mu q}^{(2)}(t, \dot{q}(t)) \epsilon^{2}$, which is an extension of Eq. (6), assuming $F_{\mu q}^{(1)}(t, 0)=F_{\mu}^{(1)}(t)$, and $F_{\mu q}^{(2)}(t, 0)=F_{\mu}^{(2)}(t)$.
Then, we write the equation of motion as: $F_{\mu q}^{(1)}(t, \dot{q}(t)) \epsilon+$ $F_{\mu q}^{(2)}(t, \dot{q}(t)) \epsilon^{2}+F_{q}\left(\partial_{t}^{3} q(t), \partial_{t}^{4} q(t), \ldots\right) \approx M_{0} \ddot{q}(t)$. Now, one can use two of the degrees of freedom of the model, namely the value of $\epsilon$ and the initial mass $M_{0}$, to simplify the problem. For instance, let us consider the changes $\epsilon \rightarrow 10^{p} \epsilon$, and $M_{0} \rightarrow 10^{2 p} M_{0}$ (with $p>0$ ) to build a new situation for which the equation of motion is $F_{\mu q}^{(1)}(t, \dot{q}(t)) \epsilon / 10^{p}+$ $F_{\mu q}^{(2)}(t, \dot{q}(t)) \epsilon^{2}+F_{q}\left(\partial_{t}^{3} q(t), \partial_{t}^{4} q(t), \ldots\right) / 10^{2 p} \approx M_{0} \ddot{q}(t)$. Increasing the value of $p$, we can inhibit the effect of the first and third terms in the last equation, so that we can set up a situation where these terms can be neglected in comparison with the second one, resulting in the approximate equation of motion $F_{\mu q}^{(2)}(t, \dot{q}(t)) \epsilon^{2} \approx M_{0} \ddot{q}(t)$. In other words, for a suitable choice of the initial mass $M_{0}$, the force $F_{\mu q}^{(2)}(t, \dot{q}(t))$ defines effectively the mean trajectory of the object. By keeping increasing the mass $M_{0}$, one can produce smaller accelerations, so that the velocities of the object are of such magnitude that the boundary condition imposed by the object on the field, considered by the tangential frame, after mapped into the boundary condition viewed by the laboratory system, can be approximately given by $F_{\mu q}^{(2)}(t, \dot{q}(t)) \approx F_{\mu q}^{(2)}(t, 0)=$ $F_{\mu}^{(2)}(t)$. With this approximation, we have the equation of motion given by $F_{\mu}^{(2)}(t) \epsilon^{2} \approx M_{0} \ddot{q}(t)$. Integrating in time, $\int_{-\infty}^{+\infty} F_{\mu}^{(2)}(t) \mathrm{d} t$, using the mentioned formula shown for $\tilde{F}_{\mu}^{(2)}(\omega)$, the property $\eta\left(\omega, \omega^{\prime}\right)=\eta\left(\omega^{\prime}, \omega\right)=\eta\left(-\omega,-\omega^{\prime}\right)$, and also considering that $\dot{q}(t<-\tau)=0$, we get

$$
v_{f} \approx-\mathcal{P} / M_{0}
$$

where $v_{f}=\dot{q}(t>\tau)$ is the mean final velocity. Note that $-\mathcal{P}$ is the opposite of the net momentum transferred from the object to the field [see Eq. (5)], so that the momentum transferred to the object, caused by the action of the force $F_{\mu}^{(2)}(t) \epsilon^{2}$, is directly correlated with the particle creation process. This situation is illustrated in Figs. 2.c and 3.a.

Let us give attention to another situation. The values of $\epsilon$ and $p$ can be chosen in such way that the term related to $F_{\mu q}^{(1)}(t, \dot{q}(t))$ becomes dominant in relation to $F_{\mu q}^{(2)}(t, \dot{q}(t)) \epsilon^{2}$. We consider [as done in a similar way for $F_{\mu q}^{(2)}(t, \dot{q}(t)) \epsilon^{2}$ ] the approximation $F_{\mu q}^{(1)}(t, \dot{q}(t)) \approx F_{\mu q}^{(1)}(t, 0)=F_{\mu}^{(1)}(t)$. It can be shown that $\int_{-\infty}^{+\infty} F_{\mu}^{(1)}(t) \mathrm{d} t=0$, which means that, although this force makes the position of the object vary in time, the total net momentum transferred to the object is null. This situation is illustrated in Fig. 3.b.

Another situation can be obtained by manipulating the values of $\epsilon$ and $p$ in such way that $F_{\mu}^{(1)}(t) \epsilon$ and $F_{\mu}^{(2)}(t) \epsilon^{2}$ have similar magnitudes. The presence of $F_{\mu}^{(1)}(t) \epsilon$ disturbs the mean trajectory but does not change the final velocity $v_{f}$ obtained if only $F_{\mu}^{(2)}(t) \epsilon^{2}$ was considered. This case is illustrated in Fig. 3.c.

Finally, we can set up the values of $\epsilon$ and $p$ so that we have to consider all terms, $F_{\mu q}^{(1)}(t) \epsilon$, $F_{\mu q}^{(2)}(t) \epsilon^{2}$ and $F_{q}\left(\partial_{t}^{3} q(t), \partial_{t}^{4} q(t), \ldots\right)$. One can see that $\int_{-\infty}^{+\infty} F_{q}\left(\partial_{t}^{3} q(t), \partial_{t}^{4} q(t), \ldots\right) \dot{q}(t) \mathrm{d} t<0$, so that the net 


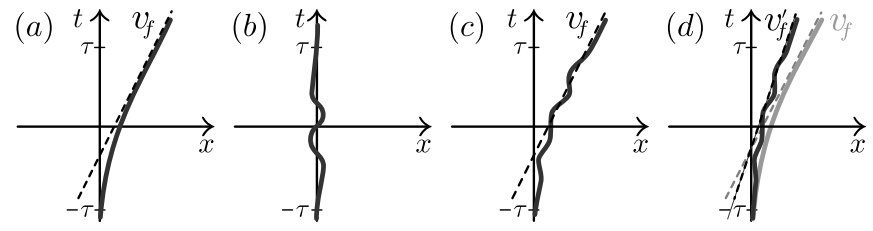

Figure 3. Mean trajectories $q(t)$ (continuous lines) of an object initially $(t<-\tau)$ at rest. (a) Illustration of $q(t)$ when just the term $F_{\mu}^{(2)}(t) \epsilon^{2}$ is considered. The mean final velocity $v_{f}$ is indicated by the dashed line. (b) Illustration of $q(t)$ when only the term $F_{\mu}^{(1)}(t) \epsilon$ is taken into account. The null contribution of this force to the mean final velocity of the object is indicated by the vertical inclination of the curve $q(t)$ for $t>\tau$. (c) Illustration of $q(t)$ when the sum $F_{\mu}^{(1)}(t) \epsilon+F_{\mu}^{(2)}(t) \epsilon^{2}$ is considered. The mean trajectory is drawn by introducing deformations on the curve shown in the case (a), but maintaining the same mean final velocity $v_{f}$. (d) Situation when all terms are considered: $F_{\mu}^{(1)}(t) \epsilon+F_{\mu}^{(2)}(t) \epsilon^{2}+F_{q}\left(\partial_{t}^{3} q(t), \partial_{t}^{4} q(t), \ldots\right)$. The net dissipation of the kinetic energy by $F_{q}\left(\partial_{t}^{3} q(t), \partial_{t}^{4} q(t), \ldots\right)$ is indicated by the mean final velocity $v_{f}^{\prime}$, with $\left|v_{f}^{\prime}\right|<\left|v_{f}\right|$. For comparison purposes, the figure also shows, in gray lines, the situation described in (a), when only $F_{\mu}^{(2)}(t) \epsilon^{2}$ was considered.

action of $F_{q}\left(\partial_{t}^{3} q(t), \partial_{t}^{4} q(t), \ldots\right)$ is to dissipate energy of the object. This leads to a mean final velocity $v_{f}^{\prime}$ with a smaller magnitude if compared with $v_{f}\left(\left|v_{f}\right|>\left|v_{f}^{\prime}\right|\right)$ obtained if only $F_{\mu}^{(1)}(t) \epsilon+F_{\mu}^{(2)}(t) \epsilon^{2}$ was considered. This situation is illustrated in Fig. 3.d.

\section{SUMMARY OF THE RESULTS AND FINAL REMARKS}

In the model proposed here, a static object, isolated from everything and just interacting with the quantum vacuum, gets in motion by exciting the vacuum. Then, the net action of the vacuum field is in favor of the motion, instead of against, as it occurs in the usual dynamical Casimir effect. This motion requires a time variation of one of the parameters $[\mu(t)]$ of the object, which couple it with the quantum vacuum field. Resisting to this change, the vacuum field extracts energy from the object, converting this energy into real particles. The motion also requires an asymmetrical vacuum excitation on each side, which can be achieved by an interaction field-object described by an asymmetric scattering matrix.

The mean force acting on the object due to $\mu(t)$ can be divided in two parts, the forces $F_{\mu}^{(1)}(t) \epsilon$ and $F_{\mu}^{(2)}(t) \epsilon^{2}$. These forces only exist (are non-null) owing to the asymmetry of the object, which means that the asymmetry is fundamental to the rise of these quantum forces.

The part of the force related to $F_{\mu}^{(1)}(t)$ is a manifestation of the disturbed vacuum field in order $\epsilon$, and the correspondent force can remove the object from the rest, but gives no contribution to the net momentum. On the other hand, the term related to $F_{\mu}^{(2)}(t)$ is a manifestation of the disturbed vacuum field in order $\epsilon^{2}$, and it is a direct consequence of the momentum transferred to the object by the created particles.

The mean forces, $F_{\mu}^{(1)}(t) \epsilon$ and $F_{\mu}^{(2)}(t) \epsilon^{2}$, described in the present paper, are quantum forces emerged from the asymmetry and time-varying properties. In conjunction with the dynamical Casimir force $F_{q}\left(\partial_{t}^{3} q(t), \partial_{t}^{4} q(t), \ldots\right)$, these three forces define, in the approximation considered here, a mean trajectory for the object. Finally, the object, starting from the rest, gets a non-null mean final velocity, so that, exciting the vacuum, it moves.

\section{ACKNOWLEDGMENTS}

D.T.A. thanks the hospitality of the Centro de Física, Universidade do Minho, Braga, Portugal.
[1] G. T. Moore, Quantum theory of the electromagnetic field in a variable-length one-dimensional cavity, J. Math. Phys. (N.Y.) 11, 2679 (1970).

[2] B. S. DeWitt, Quantum field theory in curved spacetime, Phys. Rep. 19, 295 (1975).

[3] S. A. Fulling and P. C. W. Davies, Radiation from a moving mirror in two dimensional space-time: conformal anomaly, Proc. R. Soc. A. 348, 393 (1976).

[4] P. C. W. Davies and S. A. Fulling, Radiation from moving mirrors and from black holes, Proc. R. Soc. A 356, 237 (1977).

[5] P. Candelas and D. Deutsch, On the vacuum stress induced by uniform acceleration or supporting the ether, Proc. R. Soc. A 354, 79 (1977).

[6] V. V. Dodonov, Dynamical Casimir effect: Some theoretical aspects, J. Phys. Conf. Ser. 161, 012027 (2009).

[7] V. V. Dodonov, Current status of the dynamical Casimir effect, Phys. Scr. 82, 038105 (2010).

[8] D. A. R. Dalvit, P. A. M. Neto, and F. D. Mazzitelli, Fluctuations, dissipation and the dynamical Casimir effect (Springer-
Verlag Berlin Heidelberg, 2011) Chap. 13, pp. 419-457, edited by D. A. R. Dalvit, P. Milonni, D. Roberts, and F. da Rosa.

[9] V. Dodonov, Fifty years of the dynamical Casimir effect, Physics 2, 67 (2020).

[10] W.-J. Kim, J. H. Brownell, and R. Onofrio, Detectability of dissipative motion in quantum vacuum via superradiance, Phys. Rev. Lett. 96, 200402 (2006).

[11] J. H. Brownell, W. J. Kim, and R. Onofrio, Modelling superradiant amplification of Casimir photons in very low dissipation cavities, J. Phys. A Math. Theor. 41, 164026 (2008).

[12] A. Motazedifard, A. Dalafi, M. Naderi, and R. Roknizadeh, Controllable generation of photons and phonons in a coupled Bose-Einstein condensate-optomechanical cavity via the parametric dynamical Casimir effect, Ann. Phys. (N. Y). 396, 202 (2018).

[13] M. Sanz, W. Wieczorek, S. Gröblacher, and E. Solano, Electromechanical Casimir effect, Quantum 2, 91 (2018).

[14] W. Qin, V. Macrì, A. Miranowicz, S. Savasta, and F. Nori, Emission of photon pairs by mechanical stimulation of the squeezed 
vacuum, Phys. Rev. A 100, 062501 (2019).

[15] S. Butera and I. Carusotto, Mechanical backreaction effect of the dynamical Casimir emission, Phys. Rev. A 99, 053815 (2019).

[16] E. Yablonovitch, Accelerating reference frame for electromagnetic waves in a rapidly growing plasma: Unruh-daviesfulling-dewitt radiation and the nonadiabatic casimir effect, Phys. Rev. Lett. 62, 1742 (1989).

[17] Y. E. Lozovik, V. G. Tsvetus, and E. A. Vinogradov, Femtosecond parametric excitation of electromagnetic field in a cavity, Pis'ma Zh. Éksp. Teor. Fiz. 61, 711 (1995).

[18] C. M. Wilson, G. Johansson, A. Pourkabirian, M. Simoen, J. R. Johansson, T. Duty, F. Nori, and P. Delsing, Observation of the dynamical Casimir effect in a superconducting circuit, Nature (London) 479, 376 (2011).

[19] P. Lähteenmäki, G. S. Paraoanu, J. Hassel, and P. J. Hakonen, Dynamical Casimir effect in a Josephson metamaterial, Proc. Natl. Acad. Sci. U.S.A. 110, 4234 (2013).

[20] S. Vezzoli, A. Mussot, N. Westerberg, A. Kudlinski, H. Dinparasti Saleh, A. Prain, F. Biancalana, E. Lantz, and D. Faccio, Optical analogue of the dynamical casimir effect in a dispersion-oscillating fibre, Communications Physics 2, 84 (2019).

[21] B. H. Schneider, A. Bengtsson, I. M. Svensson, T. Aref, G. Johansson, J. Bylander, and P. Delsing, Observation of broadband entanglement in microwave radiation from a single time-varying boundary condition, Phys. Rev. Lett. 124, 140503 (2020).

[22] C. Braggio, G. Bressi, G. Carugno, C. Del Noce, G. Galeazzi, A. Lombardi, A. Palmieri, G. Ruoso, and D. Zanello, A novel experimental approach for the detection of the dynamical Casimir effect, Europhys. Lett. 70, 754 (2005).

[23] A. Agnesi, C. Braggio, G. Bressi, G. Carugno, G. Galeazzi, F. Pirzio, G. Reali, G. Ruoso, and D. Zanello, MIR status report: an experiment for the measurement of the dynamical Casimir effect, J. Phys. A 41, 164024 (2008).

[24] A. Agnesi, C. Braggio, G. Bressi, G. Carugno, F. D. Valle, G. Galeazzi, G. Messineo, F. Pirzio, G. Reali, G. Ruoso, D. Scarpa, and D. Zanello, MIR: An experiment for the measurement of the dynamical Casimir effect, J. Phys. Conf. Ser. 161, 012028 (2009).

[25] J. R. Johansson, G. Johansson, C. M. Wilson, and F. Nori, Dynamical casimir effect in a superconducting coplanar waveguide, Phys. Rev. Lett. 103, 147003 (2009).

[26] F. X. Dezael and A. Lambrecht, Analogue Casimir radiation using an optical parametric oscillator, Europhys. Lett. 89, 14001 (2010).

[27] T. Kawakubo and K. Yamamoto, Photon creation in a resonant cavity with a nonstationary plasma mirror and its detection with Rydberg atoms, Phys. Rev. A 83, 013819 (2011).

[28] D. Faccio and I. Carusotto, Dynamical Casimir effect in optically modulated cavities, Europhys. Lett. 96, 24006 (2011).
[29] W. Naylor, Towards particle creation in a microwave cylindrical cavity, Phys. Rev. A 86, 023842 (2012).

[30] W. Naylor, Vacuum-excited surface plasmon polaritons, Phys. Rev. A 91, 053804 (2015).

[31] A. Motazedifard, M. H. Naderi, and R. Roknizadeh, Analogue model for controllable Casimir radiation in a nonlinear cavity with amplitude-modulated pumping: generation and quantum statistical properties, J. Opt. Soc. Am. B 32, 1555 (2015).

[32] J. M. Muñoz Castañeda and J. Mateos Guilarte, $\delta-\delta^{\prime}$ generalized Robin boundary conditions and quantum vacuum fluctuations, Phys. Rev. D 91, 025028 (2015).

[33] J. D. L. Silva, A. N. Braga, and D. T. Alves, Dynamical Casimir effect with $\delta-\delta^{\prime}$ mirrors, Phys. Rev. D 94, 105009 (2016).

[34] G. Barton and A. Calogeracos, On the quantum electrodynamics of a dispersive mirror.: I. Mass shifts, radiation, and radiative reaction, Ann. Phys. (N.Y.) 238, 227 (1995).

[35] A. Calogeracos and G. Barton, On the quantum electrodynamics of a dispersive mirror:: II. The boundary condition and the applied force via Dirac's theory of constraints, Ann. Phys. (N.Y.) 238, 268 (1995).

[36] M.-T. Jaekel and S. Reynaud, Fluctuations and dissipation for a mirror in vacuum, Quantum Opt. 4, 39 (1992).

[37] A. Lambrecht, M.-T. Jaekel, and S. Reynaud, Motion induced radiation from a vibrating cavity, Phys. Rev. Lett. 77, 615 (1996).

[38] S. Sinha and R. D. Sorkin, Brownian motion at absolute zero, Phys. Rev. B 45, 8123 (1992).

[39] M.-T. Jaekel and S. Reynaud, Quantum fluctuations of position of a mirror in vacuum, J. Phys. I (France) 3, 1 (1993).

[40] D. J. Stargen, D. Kothawala, and L. Sriramkumar, Moving mirrors and the fluctuation-dissipation theorem, Phys. Rev. D 94, 025040 (2016).

[41] Q. Wang, Z. Zhu, and W. G. Unruh, How the huge energy of quantum vacuum gravitates to drive the slow accelerating expansion of the universe, Phys. Rev. D 95, 103504 (2017).

[42] P. A. M. Neto, Vacuum radiation pressure on moving mirrors, Journal of Physics A: Mathematical and General 27, 2167 (1994).

[43] A. L. C. Rego, B. W. Mintz, C. Farina, and D. T. Alves, Inhibition of the dynamical Casimir effect with Robin boundary conditions, Phys. Rev. D 87, 045024 (2013).

[44] J. D. L. Silva, A. N. Braga, A. L. C. Rego, and D. T. Alves, Interference phenomena in the dynamical Casimir effect for a single mirror with Robin conditions, Phys. Rev. D 92, 025040 (2015).

[45] L. H. Ford and A. Vilenkin, Quantum radiation by moving mirrors, Phys. Rev. D 25, 2569 (1982).

[46] B. Mintz, C. Farina, P. A. Maia Neto, and R. B. Rodrigues, Casimir forces for moving boundaries with Robin conditions, J. Phys. A 39, 6559 (2006). 